\title{
Reply to Merrow et al. regarding additional imaging features of intramuscular capillary-type hemangioma and the importance of ultrasound
}

\author{
Gulraiz Chaudry • Sabri Yilmaz • Ahmad I. Alomari
}

Received: 18 March 2014 / Accepted: 30 March 2014 /Published online: 27 April 2014

(C) Springer-Verlag Berlin Heidelberg 2014

Sir,

We thank Merrow and colleagues [1] for their valuable comments on our recent article "Intramuscular capillary-type hemangioma: radiologic-pathologic correlation" [2]. The authors describe additional imaging findings in two patients with histologically confirmed intramuscular capillary-type hemangioma (ICTH). They demonstrated, primarily by ultrasonography, the involvement of almost the entire length of the muscle and preservation of the muscle architecture.

Indeed, we had identified fairly comparable features in 4 out of the 11 patients with sonographic studies. Nevertheless, architectural preservation of the muscle was relative, with disruption of the surrounding muscular architecture in areas of fatty infiltration. In our cohort, involvement of almost the entirety of the muscles was only noted in two patients. In fact, the lesion was resected in 5 of these 11 children due to the localized nature of the tumor.
Given the rarity of this entity, it is possible that the imaging features described by Merrow et al [1]. are suggestive of ICTH and we concur with the authors that further studies are needed to characterize this rare tumor.

Conflicts of interest None.

\section{References}

1. Merrow AC, Gupta A, Adams DM (2014) Additional imaging features of intramuscular capillary-type hemangioma: the importance of ultrasound. Pediatr Radiol. doi:10.1007/s00247-014-2989-x

2. Yilmaz S, Kozakewich HP, Alomari AI et al (2014) Intramuscular capillary-type hemangioma: radiologic-pathologic correlation. Pediatr Radiol 44:558-565
G. Chaudry $(\bowtie) \cdot$ S. Yilmaz $\cdot$ A. I. Alomari

Division of Vascular and Interventional Radiology,

Boston Children's Hospital and Harvard Medical School,

300 Longwood Ave.,

Boston, MA 02115, USA

e-mail: gulraiz.chaudry@childrens.harvard.edu 